\title{
Current-modulated optical properties of vanadium dioxide thin films in the phase transition region
}

\section{Citation}

Zhang, Shuyan, Mikhail A. Kats, Yanjie Cui, You Zhou, Yu Yao, Shriram Ramanathan, and Federico Capasso. 2014. "Current-Modulated Optical Properties of Vanadium Dioxide Thin Films in the Phase Transition Region." Applied Physics Letters 105 (21): 211104. https:// doi.org/10.1063/1.4902924.

\section{Permanent link}

http://nrs.harvard.edu/urn-3:HUL.InstRepos:41371713

\section{Terms of Use}

This article was downloaded from Harvard University's DASH repository, and is made available under the terms and conditions applicable to Other Posted Material, as set forth at http:// nrs.harvard.edu/urn-3:HUL.InstRepos:dash.current.terms-of-use\#LAA

\section{Share Your Story}

The Harvard community has made this article openly available. Please share how this access benefits you. Submit a story.

Accessibility 


\title{
Current-modulated optical properties of vanadium dioxide thin films in the phase transition region
}

\author{
Shuyan Zhang, Mikhail A. Kats, Yanjie Cui, You Zhou, Yu Yao, Shriram Ramanathan, \\ and Federico Capasso ${ }^{a)}$ \\ School of Engineering and Applied Sciences, Harvard University, Cambridge, Massachusetts 02138, USA
}

(Received 14 October 2014; accepted 16 November 2014; published online 25 November 2014)

\begin{abstract}
Vanadium dioxide $\left(\mathrm{VO}_{2}\right)$ is a correlated electron material which undergoes an insulator-metal transition proximal to room temperature. The large change of optical properties across this phase transition is promising for tunable optical and optoelectronic devices especially at infrared frequencies. We demonstrate the ability to locally tune the optical properties on the micron scale through a simple design consisting of two electrodes patterned on a $\mathrm{VO}_{2}$ thin film. By current injection between the electrodes, a localized conducting path (metallic phase) can be formed within the insulating background. The width of the conducting path can be controlled by varying the applied current. Fourier transform infrared imaging shows that this current-modulated reflectance changes significantly over a distance on the order of the wavelength in the mid-infrared spectral range. (C) 2014 AIP Publishing LLC. [http://dx.doi.org/10.1063/1.4902924]
\end{abstract}

Vanadium dioxide $\left(\mathrm{VO}_{2}\right)$ is a correlated electron material which features an insulator-metal transition (IMT). The thermally driven IMT occurs near room temperature $(\sim 340 \mathrm{~K})$ and results in an on/off resistance ratio of up to four orders of magnitude. ${ }^{1}$ This IMT also results in a large change in the optical transmittance in the near-infrared spectral range, which has found applications in energy-efficient windows ${ }^{2}$ and high-efficiency switches; ${ }^{3}$ in the infrared, a close-to-zero reflectance has been used as a perfect absorber, ${ }^{4}$ thermal emitter, ${ }^{5}$ and resonance tuning of optical antennas, ${ }^{6}$ among others. The onset of the IMT can be percolative in nature, accompanied by phase inhomogeneity, where the metallic and insulating phases coexist and various aspects of the transition are an active area of study. ${ }^{3,7-9}$ The transition can be driven electrically by current injection, and the threshold can be modulated by external stimuli including pressure and ambient temperature or by chemical doping. ${ }^{10}$

Because the metallic and insulating phases possess distinctly different electrical and optical properties, dynamic spatial control of phase inhomogeneity can be utilized to realize active metamaterials and metasurfaces, as well as other tunable optical and optoelectronic devices. Recently, $\mathrm{VO}_{2}$ based spatially tunable gradient index metasurfaces have been demonstrated in the terahertz spectral regime where the tuning was achieved by applying heat or voltage pulses to a $\mathrm{VO}_{2}$ thin film combined with split ring resonators. ${ }^{11,12}$ However, as the authors pointed out, tradeoffs exist between the degree of spatial control and the strength of the tuning of the optical properties.

In this paper, we demonstrate electrical tuning of the mid-infrared optical properties of $\mathrm{VO}_{2}$ on the micron scale. Our simple design provides dynamic control of the geometry of metallic and insulating phase regions within a compositionally homogeneous $\mathrm{VO}_{2}$ thin film. A two-terminal device consisting of a pair of wide electrodes patterned on a $\mathrm{VO}_{2}$

\footnotetext{
${ }^{\text {a) }}$ Author to whom correspondence should be addressed. Electronic mail: capasso@seas.harvard.edu.
}

thin film was fabricated. By current injection, a localized micron-scale conducting path comprising metal-phase $\mathrm{VO}_{2}$ was formed within the gap between the electrodes, surrounded by insulator-phase $\mathrm{VO}_{2}$. The spatial extent of this conducting path and thereby its optical properties can be deterministically controlled locally via the applied current which allows the development of tunable optical devices.

Our sample consists of a $\mathrm{VO}_{2}$ film grown on a polished c-plane sapphire wafer substrate by RF-magnetron sputtering with a $\mathrm{V}_{2} \mathrm{O}_{5}$ target (99:9\% purity, AJA International, Inc.). During film growth, the substrate temperature and RF source gun power were maintained at $550^{\circ} \mathrm{C}$ and $125 \mathrm{~W}$, respectively. A mixture of 99.50-sccm Ar and 0.50-sccm $\mathrm{O}_{2}$ was used as the sputtering gas while maintaining the total pressure at 10 mTorr. The film thickness is approximately $230 \mathrm{~nm}$ measured by a scanning ellipsometer (Gaertner Scientific) with a root-mean-square roughness value of approximately $3 \mathrm{~nm}$ measured by an optical profiler (Taylor Hobson CCI HD optical profiler system). The resistance on/ off ratio of the film is $R_{25}{ }^{\circ} \mathrm{C} / R_{100}{ }^{\circ} \mathrm{C}=610$. The transition temperature is $69{ }^{\circ} \mathrm{C}$ given by the average of the maximum of derivative curves $d[\log (R)] / d T$ during heating and cooling, respectively, i.e., $T_{I M T}=\left(T_{I M T}^{\text {heating }}+T_{I M T}^{\text {cooling }}\right) / 2$. The hysteresis width $\left(T_{I M T}^{\text {heating }}-T_{I M T}^{\text {cooling }}\right)$ is $4{ }^{\circ} \mathrm{C}$. The calculations are provided in the supplementary material. ${ }^{13}$

A pair of metallic electrodes were patterned on the sample using direct-write photolithography (MicroChem LOR3A, Shipley S1818 photoresist, and Heidelberg $\mu$ PG501 writer), electron beam evaporation (Denton evaporator), and lift-off. The electrode composition was $\mathrm{Ti}(10 \mathrm{~nm}) / \mathrm{Au}$ $(300 \mathrm{~nm})$. The gap between the electrodes was $15 \mu \mathrm{m}$, and the width was $300 \mu \mathrm{m}$. The sample was mounted on a probe station and characterized using a parameter analyzer (Agilent 4156C). Before all other electrical measurements, electroforming of the contacts was performed to break down any oxide layers and to form a conducting path at the Ti$\mathrm{VO}_{2}$ interface. The electroforming process involved applying a voltage to the electrodes from 0 to a value at which a sharp 

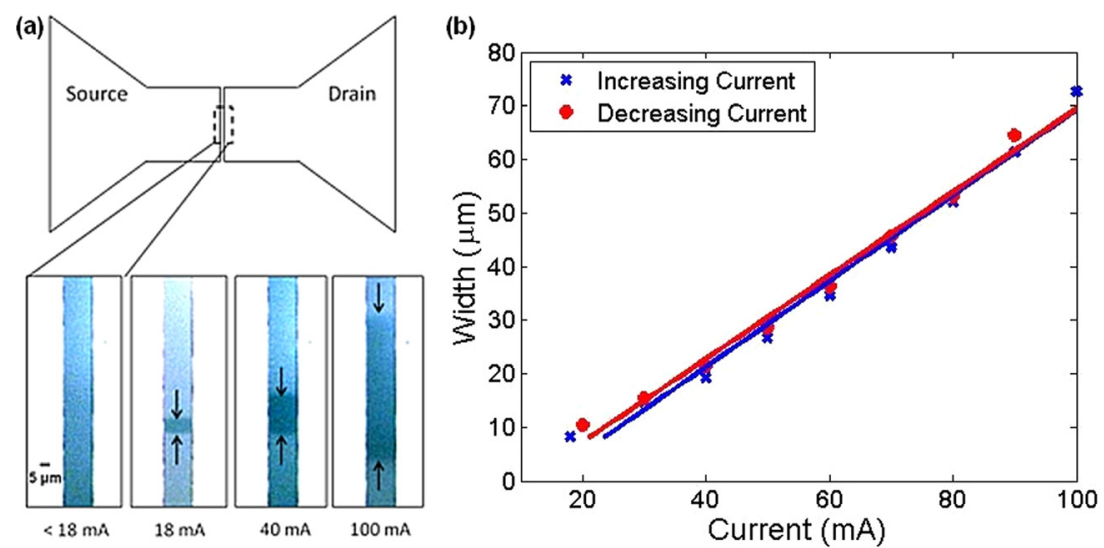

FIG. 1. Conducting path dynamics: (a) a schematic of the two-terminal device and optical microscope images zoomed in on the gap region showing a conducting path (indicated by the black arrows) appeared at a threshold current and grew wider as the current was increased (contrast of the images was increased for visual clarity). (b) Curve fit results showing a linear correlation between the width of the conducting path and the applied current in the increasing current mode (blue curve) and the decreasing current mode (red curve).

increase in conductivity was observed, and the I-V curve became $\mathrm{S}$-shaped ${ }^{14}$ (12 V in our experiment). The experiments were performed in an ambient atmosphere at room temperature with no external temperature control in the set-up. The Raman spectroscopy study was performed using a WITec Alpha300 confocal Raman microscope with a laser source of $\lambda=532 \mathrm{~nm}$. The Fourier transform infrared (FTIR) spectroscopy measurements were performed using a Bruker Vertex 70 FTIR spectrometer with a Hyperion 3000 microscope equipped with a focal plane array (FPA) detector.

Fig. 1(a) shows a schematic of the two-terminal device and optical microscope images zoomed in on the gap region (black dashed box) when the applied current was gradually increased from 0 to $100 \mathrm{~mA}$. The contrast of the images was increased for visual clarity. Below $18 \mathrm{~mA}$, the increasing current did not lead to any visually noticeable features. When the current reached $18 \mathrm{~mA}$, a dark strip (indicated by black arrows) suddenly appeared. As explained below, this dark strip corresponds to a "conducting path"-a region of metal-phase $\mathrm{VO}_{2}$ which has a much higher conductivity than the surrounding insulator-phase $\mathrm{VO}_{2}$. As the current was increased further, the width of this conducting path increased until it reached the width of the electrodes. Similarly, when the current was decreased, the width decreased as would be expected for the reversible transition. The physics behind the formation of the conducting path was discussed in Ref. 15 . We focus on the optical control that this tunable path can provide.

Fig. 1(b) shows a linear relationship (curve fit with an r-squared value close to 0.99) between the width of the conducting path and the applied current for increasing current (blue curve) and decreasing current (red curve). The slopes of the curves are constant, which means that the current density in the conducting path region is approximately constant over the range of the applied current. Once a conducting path is formed, the local resistance drops significantly, which provides a favorable path for the current flow. Most of the power is dissipated in this metal-phase region, so any additional current elevates the local temperature and hence widens the region.

Fig. 2(a) shows the I-V characteristics of the twoterminal device measured using a current source. We observed a region of negative differential resistance (NDR) from $12.5 \mathrm{~mA}$ to $91.5 \mathrm{~mA}$ for increasing current and from $80.5 \mathrm{~mA}$ to $8.5 \mathrm{~mA}$ for decreasing current, where the voltage decreased as the current was increased and vice versa. The onset of the NDR region corresponds to the onset of the IMT which is percolative in nature, i.e., the metallic and insulating phase coexist in $\mathrm{VO}_{2}{ }^{7,16}$ The difference between the NDR regions of the red curve and the blue curve is a result of the hysteresis. ${ }^{12,16}$

Since the conductance of the insulating phase region is much smaller compared to that of the metallic phase region and assuming that the borders between the metallic phase and insulating phase regions are relatively abrupt, we correlated the electrical measurements and the width of the conducting path to deduce the local resistivity of the metallic phase region. The resistivity $\rho$ is related to the resistance by $R=\rho \times l / A$, where $l$ is the gap size and $A$ is the product of the width of the conducting path and the thickness of the
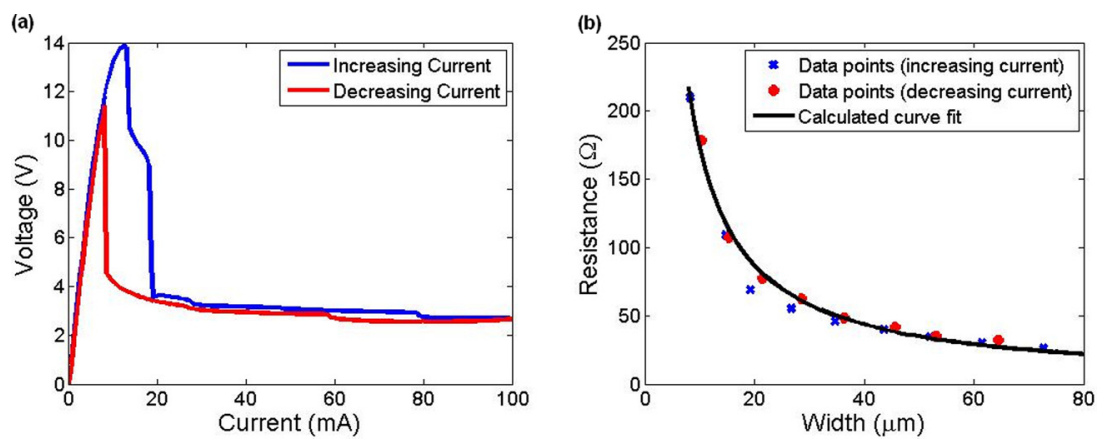

FIG. 2. (a) I-V characteristics for increasing current (blue curve) and decreasing current (red curve). (b) The measured resistance is related to the width of the conducting path.The resistivity of the metal-phase $\mathrm{VO}_{2}$ is determined to be $2.32 \times 10^{-3} \Omega \cdot \mathrm{cm}$ from the fit of the data. 
$\mathrm{VO}_{2}$ film. In Fig. 2(b), we plot the measured resistance as a function of the visually determined width of the conducting path. We fitted the data with the above expression to obtain the resistivity $\rho$ to be $2.32 \times 10^{-3} \Omega \cdot \mathrm{cm}$ with an r-squared value close to 0.99 (black curve) consistent with the literature. $^{8}$

Fig. 3(a) shows the Raman spectroscopy measurements near the conducting path region. Raman measurements are frequently used to study the structural change associated with the IMT. ${ }^{17,18}$ We compared the Raman spectrum at the center of and approximately $50 \mu \mathrm{m}$ away from the conducting path region (the latter point is outside of the frame in Fig. 3(b)). The blue curve in Fig. 3(a) is the Raman spectrum of the $\mathrm{VO}_{2}$ film with no applied current and the $\mathrm{VO}_{2}$ film was entirely in its insulating phase. At an applied current of $35 \mathrm{~mA}$, we observed a metal-phase conducting region (red curve); outside the conducting path the $\mathrm{VO}_{2}$ appeared to still be in its insulating phase (black curve). Comparing the red curve with the blue and black curves, several peaks associated with the insulating phase $\mathrm{VO}_{2}$ vanished (e.g., $196 \mathrm{~cm}^{-1}$, $224 \mathrm{~cm}^{-1}$, and $620 \mathrm{~cm}^{-1}$ ), but a broad peak at about $390 \mathrm{~cm}^{-1}$ appeared, which is a Raman active mode of the metal-phase $\mathrm{VO}_{2} \cdot{ }^{17}$

We also performed a two-dimensional scan of an area of $60 \times 60 \mu \mathrm{m}^{2}(150 \times 150$ points $)$ around the conducting region (integration time $=0.5 \mathrm{~s}$ ). We applied a sum filter which integrates the Raman signal intensity over a defined wavenumber range of interest and subtracts the background as a linear baseline from the minimum to the maximum value of the wavenumber range. The filter range selected was from $530 \mathrm{~cm}^{-1}$ to $700 \mathrm{~cm}^{-1}$ (indicated by the black box region in Fig. 3(a)) covering one of the characteristic Raman peaks of the insulator-phase $\mathrm{VO}_{2}$ at $620 \mathrm{~cm}^{-1}$. The resulting image is shown in Fig. 3(b). We can see clearly the contrast of the Raman intensities inside and outside the conducting path; the darker the color, the lower the intensity sum. The Raman intensity distribution at the edge of the conducting path shows that there is a phase co-existence region. As a result, our assumption that the edge of the conducting path is abrupt made in calculating the resistivity (Fig. 2(b)) is only approximate; nonetheless, the uncertainty is relatively small $(\sim 3 \mu \mathrm{m})$ compared to the total width of the conducting path (tens of microns).
Since both the real and imaginary parts of the refractive index change drastically as $\mathrm{VO}_{2}$ undergoes phase change, ${ }^{6}$ the optical properties are different in the conducting path region and outside of it. Fig. 4 shows mid-infrared reflectance images acquired using an FTIR microscope with an FPA detector. The FPA detectors allow for the simultaneous acquisition of 64 by 64 spatially resolved spectra, with each pixel providing an independent infrared spectrum. With a $36 \times$ objective, each pixel corresponds to an area of $1.11 \times$ $1.11 \mu \mathrm{m}^{2}$. The measurements were performed at two different currents (21 mA and $40 \mathrm{~mA}$ ). Figs. 4(a)-4(c) show the reflectance spectra at each pixel integrated over the 2.86 to $2.94 \mu \mathrm{m}$ and 8.33 to $10 \mu \mathrm{m}$ regions. The resolution of each image is diffraction limited, so the smaller the wavelength, the better the resolution. All spectra were normalized to the reflectance of the gold electrodes. The conducting path is shown at the center of the images. The line scans are taken across the middle of the conducting path indicated by the black dashed lines. The reflectance intensity sum is higher in the conducting path than outside of it. The width of the highreflectance region increased with the current. This provides a way to dynamically control the optical properties of $\mathrm{VO}_{2}$ locally and use it as a tunable optical device.

Fig. 4(d) compares the reflectance at the center of (blue), at the edge of (green), and far away from (red) the conducting path (CP) as a function of wavelength. The positions are marked as the black crosses in (b). It can be seen that the reflectance decreases gradually from a value above 0.6 at the center of the conducting path to approximately 0.4 at the edge (10 $\mu \mathrm{m}$ away from the center) and finally to approximately 0.2 far away ( $23.3 \mu \mathrm{m}$ from the center). The reflectance spectrum at the center of the conducting path increases slightly at longer wavelengths, whereas the reflectance at the edge of and far away from the conducting path decreases when the wavelength increases. This is consistent with the observations of the reflectance spectra of the same sample at temperatures above and below the transition temperature (see the supplementary material ${ }^{13}$ ). The spikes near $4.25 \mu \mathrm{m}$ and $6.25 \mu \mathrm{m}$ come from carbon dioxide and ambient water respectively which do not affect our analysis.

A $\mathrm{VO}_{2}$-based two-terminal device was fabricated and characterized by optical microscopy, electrical measurements, and Raman spectroscopy. By current injection, we
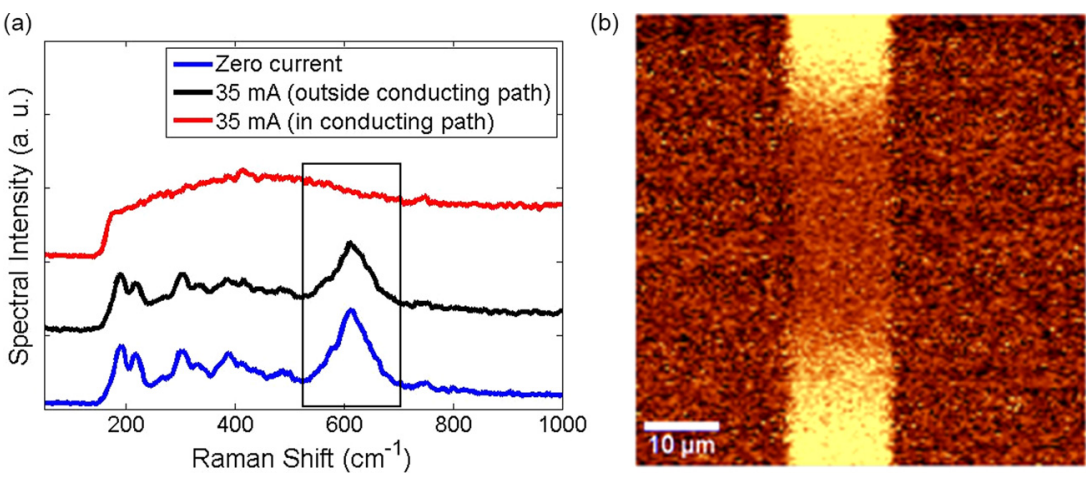

FIG. 3. (a) The blue curve is the Raman spectrum measured with zero applied current. The red and black curves are the Raman spectra measured with 35 mA applied current at the center of the conducting path and $50 \mu \mathrm{m}$ away from the conducting path, respectively. (b) Plot of the sum of the Raman signal intensity integrated over the wavenumber range from $530 \mathrm{~cm}^{-1}$ to $700 \mathrm{~cm}^{-1}$ (black box region in (a)) in an area $\left(60 \times 60 \mu \mathrm{m}^{2}\right)$ around the conducting path; the darker the color, the lower the intensity sum. 
$2.86-2.94$ microns
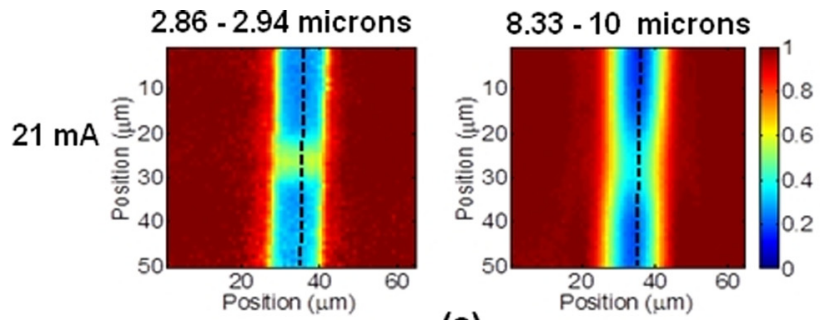

(a)
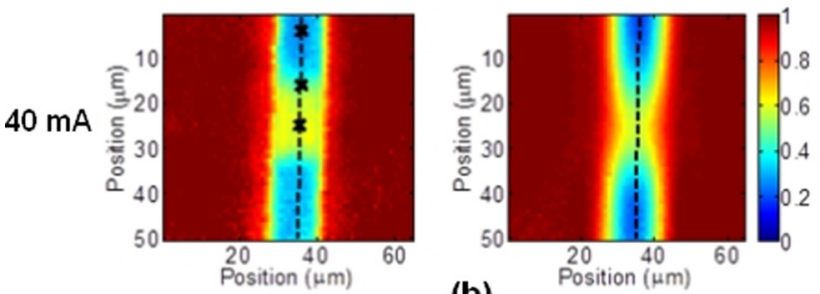

(b)
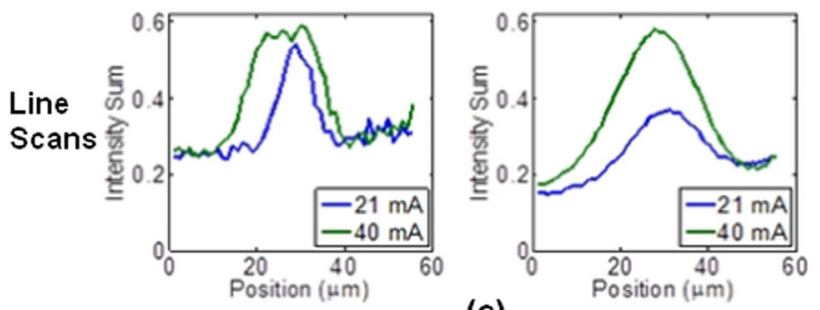

(c)

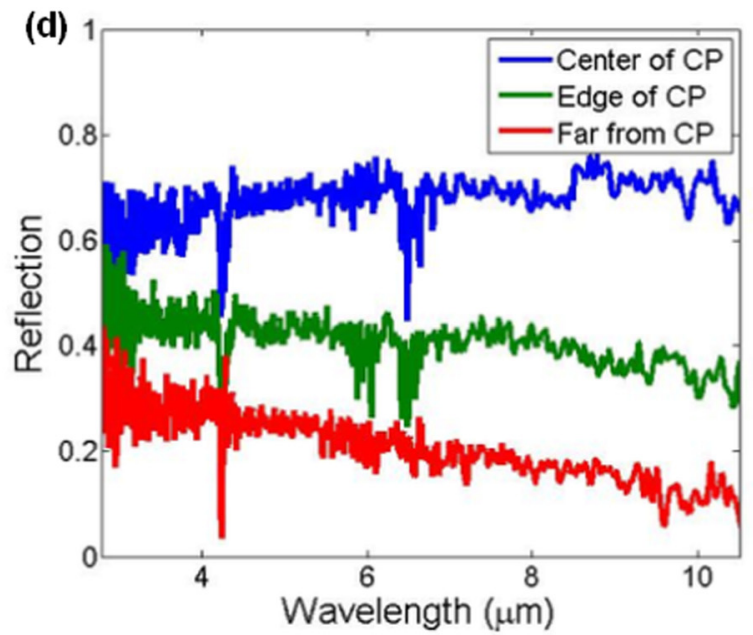

FIG. 4. (a)-(c) FTIR spectroscopy measurement at $21 \mathrm{~mA}$ and $40 \mathrm{~mA}$. The reflectance spectra are integrated over the 2.86-2.94 $\mu \mathrm{m}$ (left) and 8.33-10 $\mu \mathrm{m}$ (right) wavelength regions. (a) and (b) are the maps of the reflectance distribution after integration, and (c) shows the corresponding line scans taken at the positions indicated by the black dashed lines. (d) Infrared spectrum at the center of (blue), $10 \mu \mathrm{m}$ away from the center (green), and 23.3 $\mu \mathrm{m}$ away from (red) the conducting path (CP). The positions are shown as black crosses in (b) at $40 \mathrm{~mA}$.

are able to tune the optical properties of $\mathrm{VO}_{2}$ locally on the micron scale as observed by spatially resolved transform infrared (FTIR) measurements. The results point to interesting directions for further studies in the design of electrically tunable optical devices utilizing phase change materials.
It has previously been shown that it is possible to electrically trigger the IMT of $\mathrm{VO}_{2}$ on the nanosecond timescale. ${ }^{9}$ We envision our technique being applied to a wide range of high speed tunable structures, e.g., spatial light modulators and beam steering devices. Furthermore, if spatial control on subwavelength scales can be attained, our technique may enable a new class of dynamically tunable metamaterials and metasurfaces.

The authors would like to thank Alan She, Tobias Mansuripur, Jura Rensberg, and Steven Byrnes for their helpful discussions. This work was financially supported by AFOSR through Grant No. FA9550-12-1-0289, NSF, and by Draper Laboratory. S. Zhang acknowledges support from the Singapore A*STAR National Science Graduate Scholarship. The fabrication and some of the measurements were performed at the Center for Nanoscale Systems (CNS) at Harvard University, a member of the National Nanotechnology Infrastructure Network (NNIN). Some of the FTIR measurements were performed at the Wyss Institute at Harvard.

${ }^{1}$ F. J. Morin, Phys. Rev. Lett. 3(1), 34 (1959).

${ }^{2}$ C. Batista, R. Ribeiro, and V. Teixeira, Nanoscale Res. Lett. 6(1), 301 (2011).

${ }^{3}$ F. Guinneton, L. Sauques, J.-C. Valmalette, F. Cros, and J.-R. Gavarri, Thin Solid Films 446(2), 287 (2004).

${ }^{4}$ M. A. Kats, D. Sharma, J. Lin, P. Genevet, R. Blanchard, Z. Yang, M. M. Qazilbash, D. N. Basov, S. Ramanathan, and F. Capasso, Appl. Phys. Lett. 101(22), 22101 (2012).

${ }^{5}$ M. A. Kats, R. Blanchard, S. Zhang, P. Genevet, C. Ko, S. Ramanathan, and F. Capasso, Phys. Rev. X 3(4), 041004 (2013).

${ }^{6}$ M. A. Kats, R. Blanchard, P. Genevet, Z. Yang, M. M. Qazilbash, D. N. Basov, S. Ramanathan, and F. Capasso, Opt. Lett. 38(3), 368 (2013).

${ }^{7}$ M. M. Qazilbash, M. Brehm, B.-G. Chae, P. C. Ho, G. O. Andreev, B.-J. Kim, S. J. Yun, A. V. Balatsky, M. B. Maple, F. Keilmann, H.-T. Kim, and D. N. Basov, Science 318(5857), 1750 (2007).

${ }^{8}$ D. Ruzmetov and S. Ramanathan, in Thin Film Metal-Oxides, edited by Shriram Ramanathan (Springer, USA, 2010), p. 51.

${ }^{9}$ Y. Zhou, X. Chen, C. Ko, Z. Yang, C. Mouli, and S. Ramanathan, IEEE Electron Device Lett. 34(2), 220 (2013).

${ }^{10}$ C. N. Berglund and H. J. Guggenheim, Phys. Rev. 185(3), 1022 (1969).

${ }^{11}$ M. D. Goldflam, T. Driscoll, D. Barnas, O. Khatib, M. Royal, N. Marie Jokerst, D. R. Smith, B.-J. Kim, G. Seo, H.-T. Kim, and D. N. Basov, Appl. Phys. Lett. 102(22), 224103 (2013).

${ }^{12}$ M. D. Goldflam, T. Driscoll, B. Chapler, O. Khatib, N. M. Jokerst, S. Palit, D. R. Smith, B.-J. Kim, G. Seo, H.-T. Kim, M. D. Ventra, and D. N. Basov, Appl. Phys. Lett. 99(4), 044103 (2011).

${ }^{13}$ See supplementary material at http://dx.doi.org/10.1063/1.4902924 for details of resistance measurements and optical spectra of the device before and after the phase transition.

${ }^{14}$ F. A. Chudnovskii, L. L. Odynets, A. L. Pergament, and G. B. Stefanovich, J. Solid State Chem. 122(1), 95 (1996).

${ }^{15}$ Y. Zhao, J. Hao, C. Chen, and Z. Fan, J. Phys.: Condens. Matter 24(3), 035601 (2012).

${ }^{16}$ A. Crunteanu, J. Givernaud, J. Leroy, D. Mardivirin, C. Champeaux, J.-C. Orlianges, A. Catherinot, and P. Blondy, Sci. Technol. Adv. Mater. 11(6), 065002 (2010).

${ }^{17}$ P. Schilbe, Physica B 316-317(0), 600 (2002).

${ }^{18}$ H.-T. Kim, B.-G. Chae, D.-H. Youn, G. Kim, K.-Y. Kang, S.-J. Lee, K. Kim, and Y.-S. Lim, Appl. Phys. Lett. 86(24), 242101 (2005). 